\title{
Aprender a ser docente definiendo un modelo didáctico y unos principios de acción
}

\author{
OLGA DUARTE PIÑA \\ Departamento de Didáctica de las \\ Ciencias Experimentales y Sociales \\ Universidad de Sevilla \\ oduarte@us.es \\ ORCID: https://orcid.org/0000-0001-7341-6689 \\ D.0.I.: http://dx.doi.org/10.12795/JDU.2018.i01.86 \\ Pp.: 1532-1550
}

\section{Resumen}

Muchas veces me he preguntado si los contenidos de enseñanza del programa de una asignatura para la formación de maestros y maestras se convierten en aprendizajes útiles. A través de los distintos Ciclos de Mejora Docente y de la evaluación de los mismos he ido comprobando que el alumnado aprende los contenidos y consigue trasladarlos a experiencias de aula, pero en este aprendizaje no han incorporado de manera efectiva una reflexión crítica sobre su modelo didáctico personal ni sobre los principios que guiarían su acción futura. Con esta problemática arranca el Ciclo de Mejora en la REFID 2018. Un asunto que va más allá del tema de la asignatura, que es conocimiento didáctico patrimonial, y que constituye el fundamento de la actuación docente. 
Palabras clave: Didáctica del Patrimonio Cultural, Grado en Educación Infantil, docencia universitaria, experimentación docente universitaria, modelo didáctico personal, principios didácticos.

\section{Descripción del contexto}

El curso pasado concluía la comunicación haciendo una valoración negativa al cuestionario con el que indagué las ideas de los alumnos, pues el hecho de no poder incluir todas las preguntas en la evaluación de los aprendizajes demostraba que las excluidas dejaban de tener relevancia y podían suprimirse. También hacía una valoración positiva y afirmaba que "para enseñar bien hay que investigar sobre lo que se enseña, analizar los problemas de la práctica y las dificultades de aprendizaje del alumnado. Nada de ello se puede abordar de manera consecuente, rigurosa y justa sino es siguiendo los pasos de un ciclo de mejora." (Duarte Piña, 2017). Además, tuve la impresión de que el alumnado tomaba conciencia de los contenidos no como informaciones de un programa que obligatoriamente ha de conocer, sino como un sistema de ideas-guía para su propia vida, para su formación como ciudadanos, para su futuro como docentes. Siguiendo estas cuestiones que acabo de expresar, en el nuevo ciclo de mejora me planteé tres acciones de cambio: la primera consistió en modificar el cuestionario para indagar las ideas previas y finales del alumnado; la segunda, enseñar al alumnado a definir un modelo didáctico personal y unos principios didácticos con los que poder investigar la propia enseñanza y analizar los obstáculos y las dificultades de aprendizaje; y la tercera, modificar el programa de la asignatura para integrar la segunda acción de cambio y convertirlo en un sistema de ideas-guía.

De inicio, el curso empezó con cierta dificultad y el primer día de clase sólo tenía 15 alumnos y alumnas 
matriculados, tal situación me preocupó bastante porque la asignatura, Didáctica del Patrimonio Cultural de Andalucía que se imparte en 4o del Grado de Educación Infantil, necesita un grupo amplio para que se torne dinámica, haya una mayor diversidad de propuestas en cada uno de los grupos de trabajo y puedan compartirse la variedad experiencias de aprendizaje. Finalmente, y tras presentarse el programa y cronograma de actividades aumentó el número de inscritos hasta 27 que son los que actualmente cursan la asignatura. Después de varios años con grupos sólo de mujeres, hay dos hombres, algo que me agrada pues parece asociarse Educación Infantil a una cuestión de género.

\section{Diseño previo del Ciclo de Mejora Docente}

En el tiempo dedicado al Ciclo de Mejora Docente 2018 he planteado dos diseños encadenados. En esta comunicación se presentan ambos pero sólo los resultados del primero pues el segundo está desarrollándose aún en el momento de escribir este texto.

Para el primer diseño modifiqué el programa de la asignatura empezando por el final de la misma. Todo ello vino condicionado por una reflexión sobre ¿qué modelo de docencia formaba para una educación patrimonial deseable? Entendiendo que el conocimiento didáctico patrimonial es el núcleo principal de la asignatura, que se desarrolla en cinco unidades didácticas, es necesario que los futuros docentes tomen conciencia y se cuestionen su modelo didáctico personal. Aunque esto se planteara desde el curso 2013/2014 como cierre de los aprendizajes, en la unidad didáctica 6, no llegaba a valorar el cambio de sus ideas porque los primeros años se hacía una puesta en común grupal y a partir del curso 2016/2017 introduje una sintesis 
evaluativa individual que se añadía como última tarea en el diario personal de la asignatura.

Suponiendo que el alumnado que cursa esta asignatura ya viene de una primera experiencia práctica durante tercer año del Grado y tiene sus primeras concepciones sobre qué tipo de maestro o maestra quiere ser, me aventuré a poner la problemática de la unidad 6 (El desarrollo profesional deseable para integrar el Patrimonio Cultural de Andalucía en contextos formales y no formales) como unidad 1. A continuación se presenta el conjunto de la asignatura dividida en sus tres bloques de contenidos y el diseño de los dos núcleos problemáticos que han sido objeto de Ciclos de Mejora Docente encadenados.

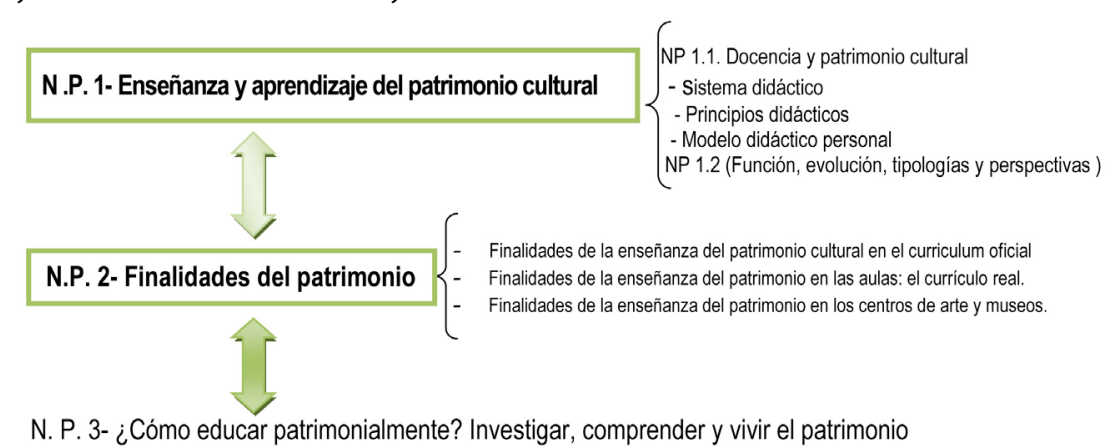

Figura 1. Bloques de contenidos de la asignatura con los dos núcleos problemáticos que se han diseñado

Jornadas de Formación e Innovación Docente del Profesorado | № 1 (2018)

(i) Esta obra se distribuye con la licencia Creative Commons Reconocimiento-NoComercial-SinObraDerivada Internacional (CC BY-NC-ND 4.0.) 


\section{¿Qué es el patrimonio cultural?}

QUÉ FUNCIÓN HA TENIDO Y DEBERIA TENER EL PATRIMONIO CULTURAL EN LA EDUCACION INFANTIL?

Figura 2. Mapa detallado del núcleo problemático 1.1, su conexión con NP1.2 y los subproblemas y contenidos asociados (Primer CMD)

Jornadas de Formación e Innovación Docente del Profesorado | № 1 (2018)

cc (i) $($ E) Esta obra se distribuye con la licencia Creative Commons 


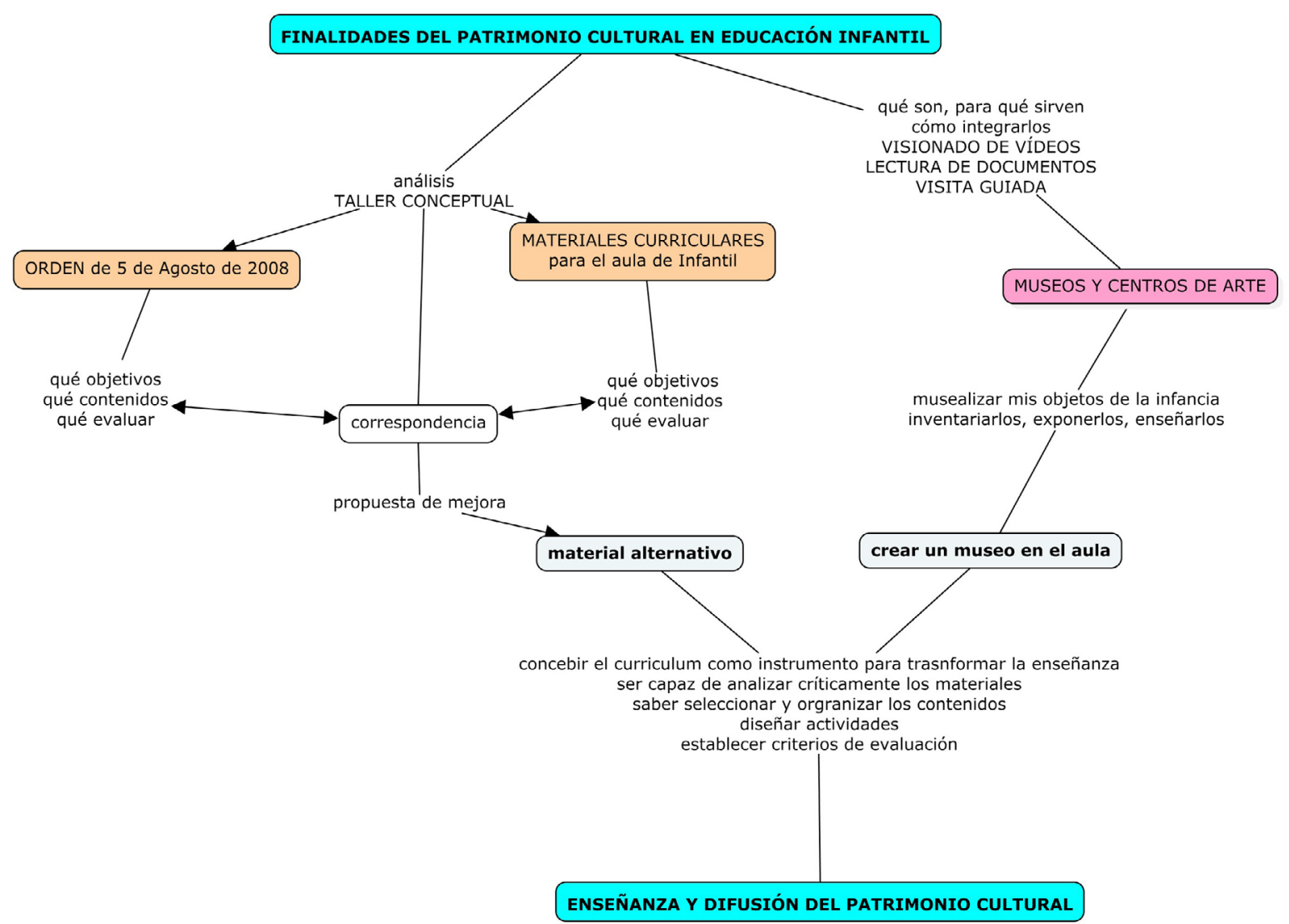

Figura 3. Mapa detallado del núcleo problemático 2, los subproblemas y contenidos asociados (Segundo CMD)

Jornadas de Formación e Innovación Docente del Profesorado | № 1 (2018)

(c) $(1) \ominus$ Esta obra se distribuye con la licencia Creative Commons

(c) $)()_{\mathrm{EY}} \mathrm{NO}_{\text {ND }}$ Reconocimiento-NoComercial-SinObraDerivada

Internacional (CC BY-NC-ND 4.0.) 
Cuestionario inicial por cada bloque de contenidos (Hipótesis iniciales estudiantes H1) $\rightarrow$ Actividades teórico-prácticas ( $\mathrm{T}+\mathrm{P}$ Actividades de ampliación y contraste) $\rightarrow$ Sesiones de puesta en común (Actividad de reformulación de ideas) Cuestionario final por cada bloque de contenidos (Hipótesis de los estudiantes H2) $\rightarrow$ Unidad didáctica y Diario personal (Hipótesis finales H3)

\section{$\mathrm{H} 1+$ Actividades $-\mathrm{H} 2+$ Síntesis evaluativa $-\mathrm{H} 3$}

Figura 4. Modelo metodológico teniendo en cuenta las mejoras que ha tenido la asignatura

\section{Tabla 1}

\section{Secuencia de las actividades Núcleo problemático 1.1}

\section{NÚCLEO PROBLEMÁTICO 1. ENSEÑANZA Y APRENDIZAJE DEL PATRIMONIO}

CULTURAL

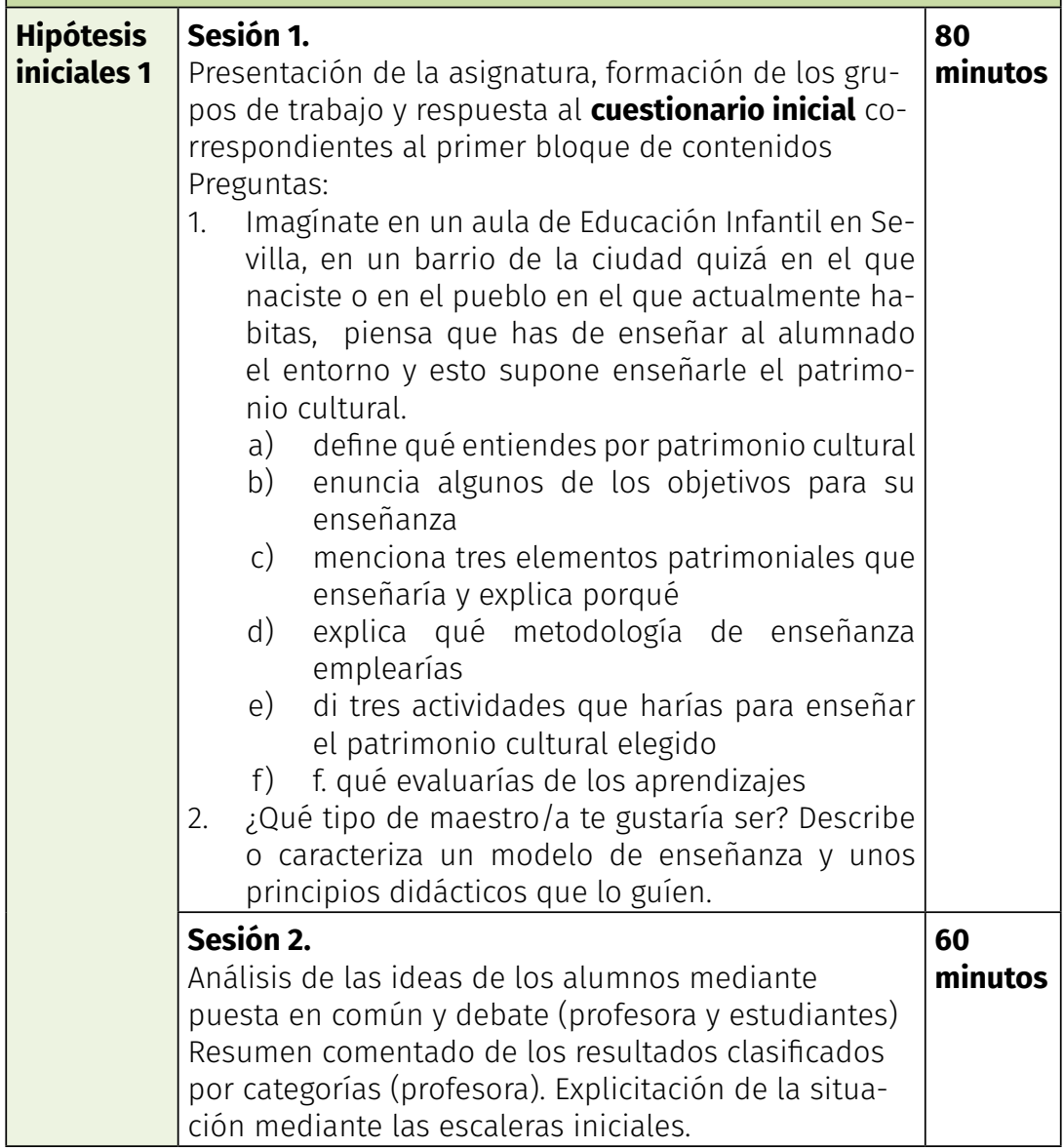




\begin{tabular}{|c|c|c|}
\hline $\begin{array}{l}\text { Actividad } \\
\text { de am- } \\
\text { pliación y } \\
\text { contraste }\end{array}$ & $\begin{array}{l}\text { Sesión 3. } \\
\text { 1. Proyección de la figura } 1 \text { "Sistema didáctico" (En- } \\
\text { señanza universitaria. Cómo mejorarla, p. 97). Re- } \\
\text { flexión por grupos de trabajo sobre los elementos } \\
\text { y sus interacciones, elaborando un documento } \\
\text { sobre la importancia que cada uno de ellos en el } \\
\text { proceso de enseñanza y aprendizaje. } \\
\text { 2. Lectura individual de los textos que aparecen en } \\
\text { el anexo: } \\
\text { DoC. 1. La metodología de enseñanza (resumen } \\
\text { del capítulo } 2 \text { del libro Enseñanza universitaria. } \\
\text { Cómo mejorarla, p.37) } \\
\text { DoC. 2. Listado de principios didácticos perso- } \\
\text { nales para definir un modelo didáctico personal } \\
\text { (Actividad 5.1 del libro Enseñanza universitaria. } \\
\text { Cómo mejorarla, pp. 94-95) } \\
\text { 3. Puesta en común y síntesis de ideas clave: el do- } \\
\text { cente debe actuar sobre todos los elementos del } \\
\text { sistema didáctico y generar sus interacciones. }\end{array}$ & $\begin{array}{l}80 \\
\text { minutos }\end{array}$ \\
\hline $\begin{array}{l}\text { Actividad } \\
\text { de reformu- } \\
\text { lación de } \\
\text { ideas }\end{array}$ & $\begin{array}{l}\text { Sesión 4. } \\
\text { 1. Intervención de dos alumnas del curso pasado } \\
\text { contando su experiencia de enseñanza y apren- } \\
\text { dizaje del patrimonio cultural a partir de la ex- } \\
\text { perimentación de dos diseños elaborados para } \\
\text { su Trabajo de Fin de Grado. Contenidos que se- } \\
\text { leccionaron y finalidades planteadas, metodolo- } \\
\text { gía empleada y actividades diseñadas, evaluación } \\
\text { (instrumentos y resultados). } \\
\text { 2. Elaboración por cada grupo de trabajo de un } \\
\text { mapa de los contenidos aprendidos, presenta- } \\
\text { ción en clase y discusión de los resultados. } \\
\text { 3. Caracterización personal de un modelo didáctico. }\end{array}$ & $\begin{array}{l}80 \\
\text { minutos }\end{array}$ \\
\hline \multirow[t]{2}{*}{$\begin{array}{l}\text { Hipótesis } \\
\text { finales } 2\end{array}$} & \multirow{2}{*}{$\begin{array}{l}\text { Sesión } \mathbf{5 .} \\
\text { Nueva respuesta a las preguntas del cuestionario } \\
\text { inicial } \\
-\quad \text { Análisis de las ideas de los alumnos me- } \\
\quad \text { diante puesta en común y debate (profesora y } \\
\quad \text { estudiantes). } \\
\text { Resumen comentado de los resultados clasificados } \\
\text { por categorías (profesora). Explicitación de la situa- } \\
\text { ción final mediante las escaleras de aprendizaje }\end{array}$} & $\begin{array}{l}60 \\
\text { minutos }\end{array}$ \\
\hline & & \begin{tabular}{|l}
20 \\
minutos
\end{tabular} \\
\hline
\end{tabular}

Jornadas de Formación e Innovación Docente del Profesorado | № 1 (2018) Esta obra se distribuye con la licencia Creative Commons Reconocimiento-NoComercial-SinObraDerivada Internacional (CC BY-NC-ND 4.0.) 
Secuencia de las actividades. Núcleo problemático 2

\begin{tabular}{|c|c|c|}
\hline \multirow[t]{2}{*}{$\begin{array}{l}\text { HIPÓTESIS } \\
\text { INICIALES } 1\end{array}$} & $\begin{array}{l}\text { Sesión 1. } \\
\text { Presentación del bloque de contenidos 2, sus nú- } \\
\text { cleos de problemas y respuesta a las preguntas del } \\
\text { cuestionario inicial correspondientes a este se- } \\
\text { gundo bloque de contenidos } \\
\text { 1. ¿Crees que en el curriculum, en los materiales } \\
\text { curriculares y en las programaciones de aula se } \\
\text { le concede la suficiente dedicación y el trata- } \\
\text { miento adecuado al patrimonio? } \\
\text { - ¿por qué? } \\
\text { 2. ¿Cómo establecer la relación entre currículum, } \\
\text { el material educativo y la programación de aula } \\
\text { para generar propuestas de conocimiento pa- } \\
\text { trimonial significativas y relevantes? } \\
\text { 3. ¿Interesa la relación entre la educación for- } \\
\text { mal y no formal para la difusión del patrimo- } \\
\text { nio cultural en la Educación Infantil? Justifica } \\
\text { la respuesta. } \\
\text { 4. ¿Llevarías a una clase de Educación Infantil (3-5 } \\
\text { años) a visitar un museo o un espacio patrimo- } \\
\text { nial? Justifica la respuesta. } \\
\text { ¿- ¿A qué museo (de Sevilla o de tu pueblo)? y } \\
\text { ¿con qué finalidad educativa? } \\
\text { NOTA: Las preguntas } 6 \text { a } 8 \text { fueron formuladas en el } \\
\text { CMD 17, la pregunta } 9 \text { se incluye nueva para este } \\
\text { ciclo de mejora. }\end{array}$ & $\begin{array}{l}80 \\
\text { minutos }\end{array}$ \\
\hline & $\begin{array}{l}\text { Sesión } \mathbf{2 .} \\
\text { - } \quad \text { Análisis de las ideas de los alumnos me- } \\
\text { diante puesta en común y debate (profesora } \\
\text { y estudiantes) } \\
\text { - } \\
\text { Resumen comentado de los resultados clasi- } \\
\text { ficados por categorías (profesora). Explicita- } \\
\text { ción de la situación mediante las escaleras } \\
\text { iniciales. }\end{array}$ & $\begin{array}{l}80 \\
\text { minutos }\end{array}$ \\
\hline
\end{tabular}




\begin{tabular}{|c|c|c|}
\hline $\begin{array}{l}\text { Actividad de } \\
\text { ampliación } \\
\text { y contraste }\end{array}$ & $\begin{array}{l}\text { Sesiones } \mathbf{3 , 4} \text { y } \mathbf{5} \\
\text { 1. Actividad: Taller conceptual 1, } 2 \text { y } 3 \text { (El guión de } \\
\text { trabajo se ofreció al alumnado) } \\
\text { Documentos de apoyo al Taller: } \\
\text { - } \quad \text { DOC. } 10 \text { - ORDEN de } 5 \text { de agosto de 2008, por la } \\
\text { que se desarrolla el currículo correspondiente } \\
\text { a la Educación Infantil en Andalucía. } \\
\text { DOC. 11- Listado de proyectos y materiales para } \\
\text { la enseñanza del patrimonio cultural. } \\
\text { DOC. 12- Guión de trabajo para analizar mate- } \\
\text { riales curriculares de Educación Infantil. }\end{array}$ & $\begin{array}{l}3 \text { ho- } \\
\text { ras y } 60 \\
\text { minutos }\end{array}$ \\
\hline $\begin{array}{l}\text { Actividad } \\
\text { de reformu- } \\
\text { lación de } \\
\text { ideas }\end{array}$ & $\begin{array}{l}\text { Sesión } \mathbf{6} \\
\text { 1. Presentación de los resultados del trabajo y va- } \\
\text { loración crítica de los resultados de cada grupo } \\
\text { de trabajo en el Diario de la asignatura. }\end{array}$ & $\begin{array}{l}80 \\
\text { minutos }\end{array}$ \\
\hline
\end{tabular}

Jornadas de Formación e Innovación Docente del Profesorado I № 1 (2018) Esta obra se distribuye con la licencia Creative Commons 
Actividad de ampliación y contraste

\section{Sesión 7}

1. ¿Qué es un museo? ¿Para qué sirven? ¿Cómo integrarlos en una didáctica del patrimonio cultural deseable?

Enlace a vídeos:

Qué es un museo: http://www.museodezaragoza.es/publicaciones/materiales-didacticos/ que-es-un-museo-cuaderno-didactico/

Tipos de museos: http://psicopeeduc.blogspot. com.es/2012/06/tipos-de-museos.html

\section{Cómo integrarlos:}

a) Sobre jóvenes y museos: https://www.youtube.com/watch?v=uxFFKU4T5Jk

b) Ejemplo de pedagogía dialógica como medio - visita al Castillo de Guzmán https:// www.youtube.com/watch?v=RcfJ2r23bKc

c) Ejemplo de pedagogía dialógica como fin proyecto Nosotras, Museo Thyssen https:// www.youtube.com/watch?v=EtKCCVjxFF8

d) Crear un museo: http://www.youtube.com/ watch?v=LkWxietaGg8

1. a. Debate de ideas sobre los visionados en grupo clase.

\section{Sesión 8}

1. Lectura de documentos:

- DOC. 13- HUERTA, R. (2010). Las maestras, los museos y el autobús. Aula de Innovación Educativa 196, 7-10.

- DOC. 14- ZAVALA, M. (2006). El museo de los niños. El Museo Anima. Revista de los Museos de Andalucía mus-A, IV (6), 32-35.

- DOC. 15- ÁlVAREZ dOMíngueZ, P. (2011). La creación de un museo del bebé. Cuadernos de Pedagogía [versión digital] 413; documento de 6 págs.

- DOC. 16- ÁVILA RUIZ, R. Mạ y DUARTE PIÑA, O. (2014). El museo infantil. Una propuesta de sensibilización del patrimonio cultural en la formación inicial del profesorado.

*Cada alumno/a elegirá un texto que ha de leer y del que extraer las ideas clave. Luego en grupo de trabajo por artículo se hará una puesta en común elaborando un documento de sintesis que ha de presentarse al grupo clase. 


\begin{tabular}{|c|c|c|}
\hline $\begin{array}{l}\text { Actividad de } \\
\text { ampliación } \\
\text { y contrastre }\end{array}$ & $\begin{array}{l}\text { Sesión } 9 \\
\text { 3. ¿Cómo se difunde el patrimonio cultural en el } \\
\text { ámbito no formal? Ruta botánica por el Parque de } \\
\text { María Luisa y Taller "Jardín de Otoño" organizados } \\
\text { por Encarte Producciones. La finalidad de esa acti- } \\
\text { vidad es que el alumnado conozca qué propuestas } \\
\text { hay para la difusión del patrimonio cultural desde } \\
\text { empresas dedicadas a ello, qué tratamiento hacen } \\
\text { de los contenidos, qué metodologías aplican, qué } \\
\text { actividades realizan y puedan ellos/as experimen- } \\
\text { tarlas desde la perspectiva docente y del alum- } \\
\text { nado de Infantil. }\end{array}$ & $\begin{array}{l}120 \\
\text { minutos }\end{array}$ \\
\hline $\begin{array}{l}\text { Actividad } \\
\text { de reformu- } \\
\text { lación de } \\
\text { ideas }\end{array}$ & 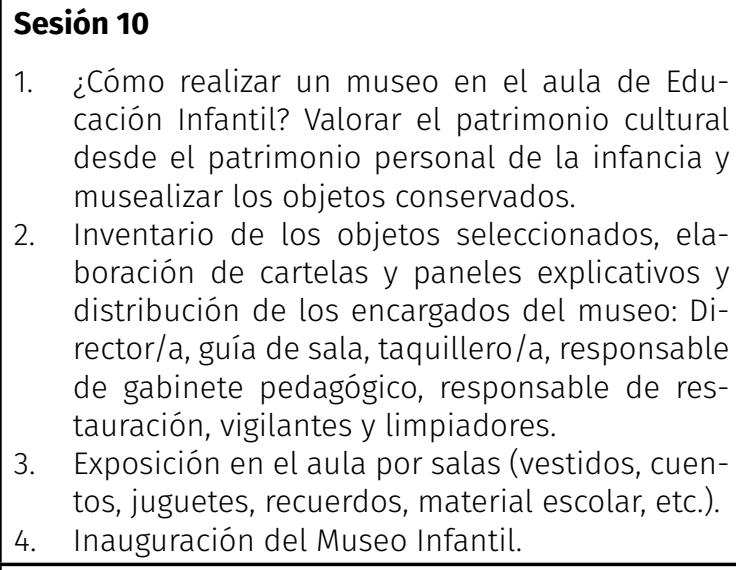 & $\begin{array}{l}160 \\
\text { minutos }\end{array}$ \\
\hline $\begin{array}{l}\text { HIPÓTESIS } \\
\text { FINALES } 2\end{array}$ & $\begin{array}{l}\text { Nueva respuesta a las preguntas del cuestionario } \\
\text { inicial } \\
\text { - Análisis de las ideas de los alumnos me- } \\
\text { diante puesta en común y debate (profesora } \\
\text { y estudiantes). } \\
\text { Resumen comentado de los resultados clasifi- } \\
\text { cados por categorías (profesora). Explicitación } \\
\text { de la situación final mediante las escaleras de } \\
\text { aprendizaje. }\end{array}$ & $\begin{array}{l}80 \\
\text { minutos }\end{array}$ \\
\hline
\end{tabular}

\section{Aplicación del Ciclo de Mejora Docente}

Puedo decir que la aplicación del primer ciclo ha sido buena y todo lo previsto ha podido realizarse. No he tenido dificultad con el alumnado que ha ido realizando las actividades programadas. Aún así, una vez analizados los 
cuestionarios iniciales y finales del núcleo problemático 1 debo entrar en determinadas valoraciones que son necesarias para ir avanzando en la asignatura y en el reto de este curso.

A medida que pasan los años percibo que el alumnado tiene más experiencias patrimoniales no sólo en su etapa escolar, también en sus prácticas como maestro/a o por la difusión que hoy en día se da en las ciudades y pueblos al patrimonio que poseen. Ello no es óbice para que se siga valorando el patrimonio histórico-artístico como el principal representante de la diversidad patrimonial y que, para la mayoría del alumnado, la perspectiva patrimonial sea estética y no simbólico-identitaria.

La sesión 3 que aparece en la Tabla 1, dedicada a trabajar el modelo didáctico y los principios, les llevó a reflexionar sobre su experiencia en las prácticas del curso pasado y a contarla individualmente por escrito, destacando la metodología de enseñanza y explicando unos principios con los que más de identificaban. Por un lado, el relato de la experiencia y su metodología me permitió constatar la escasa capacidad para conceptualizar su actuación en el aula; por otro, al comprobar que la gran mayoría elegía los principios de una enseñanza alternativa, percibí que el alumnado quiere aprender otras formas de enseñanza diferentes a las que se enfrentan, tanto en la facultad como en las aulas de Infantil, aunque no tiene desarrollado aún un pensamiento crítico y fundamentado, y reproduce fórmulas estereotipadas o usa conceptos dados por válidos, pero que no sabe realmente su utilidad y aplicación. Todo ello se complica cuando un modelo metodológico y unos principios han de vincularse con una didáctica patrimonial a la que no están acostumbrados.

Cuando les planteé en la cuarta sesión teórica que reflexionaran por grupos, a partir de lo ya trabajado 
individualmente en la sesión 3, sobre las problemas y perspectivas para mejorar la enseñanza y el aprendizaje del patrimonio cultural y respondieran a la pregunta que daba título a la unidad 1 ¿Cómo ha de ser la enseñanza y el aprendizaje del patrimonio cultural en Educación Infantil?, descubrí que la actividad de lectura individual de los textos [DOC. 1. La metodología de enseñanza (resumen del capítulo 2 del libro Enseñanza universitaria. Cómo mejorarla, p.37) y DOC. 2. Listado de principios didácticos personales para definir un modelo didáctico personal (Actividad 5.1 del libro Enseñanza universitaria. Cómo mejorarla, pp. 94-95)] no había provocado demasiado cambio en sus concepciones. Supongo que esto ha ocurrido porque los principios alternativos, enunciados como finalidades, contenidos, metodología, actividades y evaluación, ya estaban formulados en el documento que les entregué. Aunque tuvieran que explicar porqué los seleccionaban, ellos no los habían expresado desde sus propias concepciones. Por este motivo, para el curso próximo serán ellos los que expresen sus principios sobre objetivos, contenidos, metodología, actividades y evaluación, contrastándolos luego con formulaciones tradicionales y alternativas. Pero la asignatura ha seguido su curso y en la unidad 2 se trabajaron textos sobre la relación entre patrimonio y educación y la integración del patrimonio cultural en la Educación Infantil. La actividad de cierre del Bloque 1 de contenidos, que ha sido grupal y se ha desarrollado en un lugar elegido por el grupo (un pueblo o un barrio), les ha permitido acercarse a elementos patrimoniales y proponer una actividad con lo que, pasado el cuestionario de ideas finales, compruebo que los elementos del sistema didáctico (finalidades, contenidos, metodología-actividades y evaluación), que tratamos en la unidad 1 , se han enriquecido significativamente, aunque no todos. Veremos, más abajo, en la Figura 5, los cambios de toda esta problemática analizada. 
Aún así, queda recorrido en la asignatura y en el bloque 3 tendrán que elaborar, por grupos de trabajo, una propuesta de educación patrimonial que será un paso más en la construcción del conocimiento didáctico patrimonial y en los elementos del sistema didáctico.

El bloque 2 de contenidos en el que estamos actualmente, empieza por el "Taller conceptual" (Finkel, 2008) que aparece en la Tabla 2 como sesiones 3, 4 y 5. Resalto que para el diseño del segundo ciclo de mejora, se simplificó el guión de trabajo haciéndolo más compresible y dinámico, con lo que las sesiones previstas de trabajo se están cumpliendo, algo muy diferente a lo que ocurrió en el curso pasado que tuvieron que ampliarse a seis de las tres previstas. Además, se ha incluido como leiv motif, y guía del Taller, la imagen que ilustra el sistema didáctico ya referenciada más arriba. Esta imagen también guiará la unidad 5 del bloque 3 de contenidos, última de la asignatura, donde cada grupo hará y presentará el diseño de una propuesta de enseñanza patrimonial.

Jornadas de Formación e Innovación Docente del Profesorado | № 1 (2018) Esta obra se distribuye con la licencia Creative Commons 

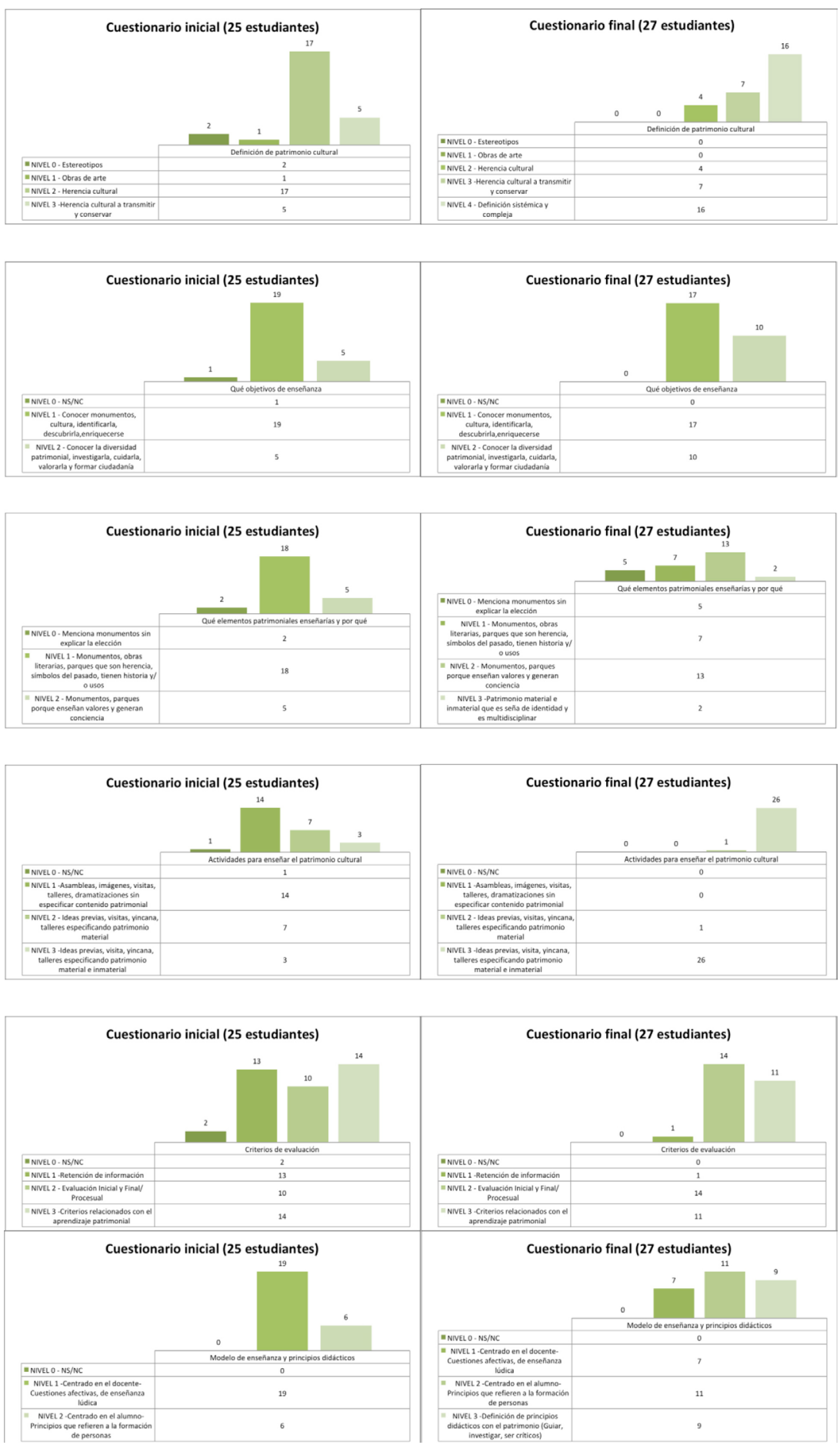

Figura 5. Escaleras de aprendizaje y evaluación

Jornadas de Formación e Innovación Docente del Profesorado | № 1 (2018) (c) (i) $\odot$ Esta obra se distribuye con la licencia Creative Commons 


\section{Evaluación del Ciclo de Mejora Docente}

El próximo ciclo de mejora mantendrá el cambio diseñado este curso y el cuestionario elaborado para los dos núcleos de contenidos pero prepararé actividades de ampliación, contraste y reformulación de ideas que desarrollen las concepciones iniciales sobre el modelo didáctico personal y los principios de acción, ideas que han de ser consensuadas en el grupo de trabajo y tenidas en cuenta en la unidad 5 y para la actividad final de la asignatura que consiste en la elaboración de una propuesta de enseñanza y aprendizaje del patrimonio cultural.

No es fácil hacer pensar al alumnado cuando lleva años siendo educado en un sistema en el que prima una enseñanza centrada en el docente, sistema del que van tomando sus propios principios de acción y modelos didácticos. Este curso, les dije desde el principio que no pensaran como alumnos sino que pensaran ya como maestros y que cada contenido que aprendieran en la asignatura lo aprehendieran para darle utilidad. Con este fin también seleccionaré los contenidos de la asignatura.

El acto de enseñar une la multiplicidad del tiempo, un tiempo en el que el estudiante se enfrenta a su pasado, vive el presente en el que se está formando y a la par va proyectando su futuro.

Principios didácticos que vienen de la experiencia anterior (Duarte Piña, 2017):

- La didáctica del patrimonio cultural ha de enseñar a enseñar y debe introducir al alumnado en la investigación sobre su docencia y los problemas de la práctica. Estos han de ser contenidos incorporados en la asignatura.

- El alumnado ha de aprender que debe investigar sobre lo que va a enseñar. 
- Lo que se enseña tiene que tener una vinculación con los intereses del alumnado o ha de procurarse que se susciten dichos intereses.

- Las actividades que se realicen en la formación de maestros deben ser potenciales actividades en un aula de Educación Infantil.

- La creatividad debe estar presente a la hora de enseñar y aprender y ha de evidenciarse.

- El futuro maestro debería desarrollar ideas sobre su modelo metodológico personal.

Principios didácticos que surgen tras esta experiencia:

- Las ideas de los maestro/as en formación he de trabajarlas constantemente para mejorar el proceso de enseñanza y aprendizaje y estas ideas he de someterlas a análisis en el aula, tanto las iniciales como las finales.

- Que el alumnado tome conciencia de los contenidos no como informaciones de un programa que obligatoriamente ha de conocer, sino como un sistema de ideas-guía para su propia vida, para su formación como ciudadanos, para su futuro como docentes.

- El futuro maestro tiene que desarrollar un modelo docente personal y unos principios didácticos que guien su conocimiento patrimonial.

- La creatividad ha de tornarse en imaginación pedagógica. La idea de imaginación pedagógica la tomo de Fernando Hernández pues perfila mi idea de creatividad docente. 


\section{Referencias bibliográficas}

Duarte Piña, O. (2017). Cómo mejorar la enseñanza de las didáctica del patrimonio cultural desde el diseño de un ciclo de mejora. IV Jornadas de Formación e Innovación Docente de la Universidad de Sevilla, pp. 734-747. ISBN: 978-84-86849-10-8

Finkel, D. (2008). Dar clases con la boca cerrada. Valencia: PÚV.

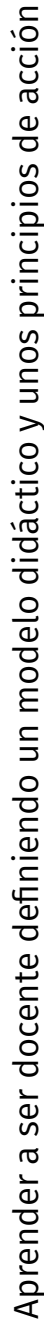

Porlán, R. (2008). El diario de clase y el análisis de la práctica. http://hdl.handle.net/11441/25448 (consultado 12 de noviembre de 2018).

Porlán, R. (coord.) (2017). Enseñanza universitaria. Cómo Mejorarla Madrid: Morata.

Jornadas de Formación e Innovación Docente del Profesorado | № 1 (2018) Esta obra se distribuye con la licencia Creative Commons 